\title{
Periconceptional Undernutrition Alters Growth Trajectory and Metabolic and Endocrine Responses to Fasting in Late-Gestation Fetal Sheep
}

\author{
MARK H. OLIVER, PAUL HAWKINS, AND JANE E. HARDING \\ Liggins Institute, University of Auckland, 92019, Auckland, New Zealand
}

\begin{abstract}
Maternal undernutrition during pregnancy can lead to impaired metabolic regulation in postnatal offspring. The extent to which such abnormalities are determined in early gestation, and may already be present in fetal life, is unknown. We studied the effect of periconceptional undernutrition on fetal growth and metabolism in late gestation. Ewes were either well fed throughout pregnancy $(\mathrm{N})$ or undernourished to reduce ewe weight by $15 \%$ from $61 \mathrm{~d}$ before until $30 \mathrm{~d}$ after mating (UN). At $121 \mathrm{~d}$, one cohort of ewes and their singleton fetuses (N, $n=10$; UN, $n=$ 10) were fasted for $72 \mathrm{~h}$, given $25 \mathrm{~g}$ of glucose i.v. over $8 \mathrm{~h}$, and refed. Fetal and placental weights were not different at $131 \mathrm{~d}$. UN ewes had higher glucose, amino nitrogen, and $\mathrm{Hb}$ concentrations than $\mathrm{N}$ ewes. UN fetuses had higher glucose and lactate, and lower amino nitrogen and $\mathrm{O}_{2}$ concentrations and $\mathrm{pH}$. UN ewes had higher insulin and lower IGF-I concentrations, and UN ewes and fetuses had higher placental lactogen concentrations. A
\end{abstract}

ABSTRACT

second cohort (N, $n=8$; UN, $n=10)$ were studied until term. Chest girth increment was reduced in UN fetuses until delivery, but birth weights were not significantly reduced. These findings suggest that fetal growth, metabolic and endocrine status, and placental function in late gestation are influenced by maternal nutrition in the periconceptional period, independent of fetal size. Metabolic and endocrine adaptations in the mother may mediate some of these effects. Such adaptations may allow continued fetal growth and survival in the face of a potentially adverse nutritional environment. (Pediatr Res 57: 591-598, 2005)

\section{IGFBP, IGF binding protein}

\section{Abbreviations}

$\mathbf{N}$, normal nutrition

PL, placental lactogen

$\mathbf{U N}$, undernutrition
Small size at birth has been associated with an increased risk of adult-onset diseases such as diabetes and cardiovascular disease (1). Although many experimental approaches have been used to investigate this relationship by inducing small fetal or birth size (2-4), maternal undernutrition throughout or only in late pregnancy has been most frequently used (4-9). Few of these studies have investigated the timing of the alterations in metabolic and endocrine function in the offspring that give rise to the increased disease risk in adulthood. Such alterations may arise in fetal life, during or shortly after the period of undernutrition, or may be an entirely postnatal phenomenon in response to later environmental or developmental stimuli.

Furthermore, epidemiologic studies of the offspring of the Dutch famine survivors showed that exposure to famine around the time of conception was at least as important as exposure in

Received February 6, 2004; accepted August 30, 2004.

Correspondence: Mark H. Oliver, Ph.D., Liggins Institute, Faculty of Medicine and Health Science, University of Auckland, Private Bag 92019, Auckland, New Zealand; e-mail: m.oliver@auckland.ac.nz

This work was funded by the Health Research Council of New Zealand.

DOI: 10.1203/01.PDR.0000155942.18096.9C late gestation in predisposing to metabolic and cardiovascular disorders during adult life, despite having little effect on birth weight $(10,11)$. Once again, the timing of the altered pathophysiology leading to these disorders is not clear. In rats, a low-protein diet only in the period up to implantation results in hypertension in the offspring (12). Embryo culture studies in sheep also suggest that altered exposure to pregnancy hormones in the immediate postfertilization period can have marked effects on subsequent fetal growth (13).

We have previously shown that maternal undernutrition in the periconceptional period alters several aspects of endocrine regulation in the late-gestation sheep fetus, with little effect on fetal size, although size at birth has not been determined. Regulation of the IGF axis is altered (14), and maturation of the insulin and hypothalamic-pituitary-adrenal (HPA) axes appears to be accelerated $(15,16)$. Others have shown that maternal undernutrition in early to mid-gestation influences aspects of endocrine and cardiovascular function in the lategestation fetal sheep $(17,18)$.

We have also shown that maternal undernutrition in the periconceptional period in sheep alters both the trajectory of fetal growth in late gestation, and also fetal growth responses 
to maternal undernutrition in late gestation (19). Fetuses whose mothers were well nourished in the periconceptional period grew rapidly in late gestation, and slowed their growth in response to late gestation maternal undernutrition. In contrast, fetuses whose mothers were undernourished during the periconceptional period grew more slowly in late gestation, but continued this slow growth during maternal undernutrition (19), although growth trajectory was not measured after $125 \mathrm{~d}$. We hypothesized that this continued growth during a lategestation nutritional insult was the result of a series of adaptations in the fetus, presumably metabolic and/or endocrine in nature, "programmed" by exposure to periconceptional undernutrition. Such adaptations would potentially allow continued fetal growth and survival in the face of nutritional limitation in later pregnancy.

We tested this hypothesis in the current study by determining the metabolic and endocrine responses of fetal sheep to a late-gestation nutritional insult in animals whose mothers had been either well nourished or undernourished around the time of conception. A second cohort of fetal sheep were subjected to the same periconceptional manipulation but then studied until term to further characterize their patterns of growth over the last 3 wk of gestation.

\section{METHODS}

Two cohorts of pregnant ewes and fetuses were studied. Breeding and nutritional manipulation was undertaken in the same way at the same time in cohorts. Cohort 1 was used to determine 1) fetal response to a late-gestation nutritional insult and 2) fetal size in late gestation. The nutritional insult (72-h food withdrawal) was the same as we have used previously to examine the control of endocrine regulators of fetal growth in late gestation $(20,21)$. Cohort 2 was used to determine 1) fetal growth trajectory all the way to delivery and 2 ) the effects of periconceptional nutrition on gestation length and size at birth. To avoid any disturbance to the fetus that might alter these outcomes, after catheterization these fetuses were not subject to any nutritional insult or other physiologic challenge in late gestation. To help ensure catheters functioned over a prolonged period until delivery, fetuses were catheterized a week later in this cohort.

Animal studies. Five-year-old Romney ewes were acclimatized for a week to indoor conditions and feeding on a concentrate diet. The pelleted diet consisted of $65 \%$ lucerne, $30 \%$ barley, with the remainder consisting of limestone, molasses, and trace element supplements. Ewes were weighed $61 \mathrm{~d}$ before mating and then randomly assigned to maintenance feeding $(\mathrm{N}$, concentrates at 3-4\% of body weight/d) or low-plane feeding (UN, fasted for $2 \mathrm{~d}$ then fed concentrates at $1-2 \%$ of body weight/d). Ewes were weighed weekly and rations for UN ewes were adjusted individually to maintain a $10-15 \%$ reduction in body weight from baseline values. Feeding levels used throughout pregnancy were based on our previous experience with this breed of sheep and conform to accepted nutritional requirements during feedlot-based experimentation (22). A fortnight before mating, the estrous cycles of ewes were synchronized with intravaginal devices containing progesterone (23). The feed restriction of $\mathrm{UN}$ ewes continued until $30 \mathrm{~d}$ after mating. Thereafter, all ewes were kept on the maintenance level of feeding. Ewes were ultrasound scanned $62 \mathrm{~d}$ after mating and only singleton-bearing ewes were used for experiments.

Cohort 1. Pregnant ewes were delivered to the laboratory at $105 \mathrm{~d}$ gestation and were instrumented at $112 \mathrm{~d}$. Under general anesthesia, polyvinyl catheters were inserted into the tarsal vein and artery of each fetal hind limb and exteriorized through the maternal flank. After closure of the uterus and maternal abdomen, catheters were inserted into a maternal carotid artery, jugular vein, and a femoral artery and vein. Antibiotics were given to ewes before surgery ( $5 \mathrm{~mL}$ Streptopen, Pitman-Moore, Wellington, New Zealand) and into the amniotic fluid before closure of the membranes and uterus $(80 \mathrm{mg}$ gentamicin, Roussel, Auckland, New Zealand). Fetuses received $80 \mathrm{mg}$ gentamicin and $600 \mathrm{mg}$ penicillin i.v. for $3 \mathrm{~d}$ after surgery (Bioheme, Vienna, Austria). All vascular catheters were flushed at least every second day until experiments commenced. At $121 \mathrm{~d}$ gestation, blood samples were collected from ewes and their fetuses $(\mathrm{N}, n=10 ; \mathrm{UN}, n=10)$ between 0800 and $0900 \mathrm{~h}$. Food was then removed and paired maternal and fetal blood samples were collected twice daily during $72 \mathrm{~h}$ of maternal fasting. At $72 \mathrm{~h}$, ewes were refed and also received $25 \mathrm{~g}$ of glucose over $8 \mathrm{~h}$ by intravenous infusion, aiming to restore maternal and hence fetal blood glucose concentrations as rapidly as possible in a "square wave" pattern. Further blood samples were collected 2, 4, 6, and $8 \mathrm{~h}$ after the start of the glucose infusion, and at 24 and $48 \mathrm{~h}$ of refeeding. Heparinized blood samples from both cohorts were centrifuged for $10 \mathrm{~min}$ at $3000 \mathrm{~g}$ and $4{ }^{\circ} \mathrm{C}$ and plasma aliquots stored at $-20^{\circ} \mathrm{C}$ until analysis. Samples for blood gas analysis were also collected on ice and analyzed within $15 \mathrm{~min}$. Ewes and their fetuses were killed by an overdose of pentobarbitone at $131 \mathrm{~d}$ gestation.

Cohort 2. Ewes were transported into the laboratory at $113 \mathrm{~d}$ gestation and acclimatized for $7 \mathrm{~d}$ before surgery. Fetal and maternal catheters were placed under general anesthesia as in cohort 1 . In addition, growth catheters were fitted around the chest of the fetus $(24,25)$. Fetal chest girth increments were recorded from the growth catheters twice daily until term $(\mathrm{N}, n=8$; $\mathrm{UN}, n=$ 10). Premature delivery was defined as birth more than 2 SD earlier than the mean gestation length for the flock.

All experiments were approved by the institutional animal ethics committee.

Biochemical analyses. Plasma glucose (26) and urea (27) concentrations were measured by standard enzymatic colorimetric methods modified for assay using a 96-well plate reader (28). Plasma lactate concentrations were measured by colorimetric assay utilizing the enzyme lactate dehydrogenase and the reduction of NAD. Plasma amino nitrogen concentrations were measured by a colorimetric assay utilizing the reaction with $\beta$-naphthoquinone sulfonate (29).

Plasma hormone concentrations were measured by specific RIA established and validated for maternal and fetal sheep plasma. Plasma insulin was measured according to previously published methods (30) except that ovine insulin (Sigma Chemical, St. Louis, MO, batch \# I9254) was used as the standard. The standard curve displaced in parallel with ovine plasma samples and crossreactivity with IGF-I or IGF-II was $<0.01 \%$. The minimal detectable concentration was $40 \mathrm{pg} / \mathrm{mL}$ plasma and the inter- and intra-assay coefficients of variation were $11.1 \%$ and $6.7 \%$, respectively. Plasma IGF-I was measured using a IGFBP-blocked RIA $(31,32)$. The detection limit was $0.7 \mathrm{ng} / \mathrm{mL}$ and the inter- and intra-assay coefficients of variation were $<10 \%$ and $<5 \%$, respectively. IGFBP1 was measured by homologous RIA (14). The assay sensitivity was $0.1 \mathrm{ng} / \mathrm{mL}$ and inter- and intra-assay coefficients of variation were $7.1 \%$ and $4.0 \%$, respectively. Plasma ovine placental lactogen (oPL) was measured by RIA as previously described (20). The minimal detectable dose was $0.05 \mathrm{ng} / \mathrm{mL}$ and inter- and intra-assay coefficients of variation were 9.7 and $5.1 \%$, respectively.

Data analysis. All data are presented as mean \pm SEM. Differences in baseline endocrine and metabolic data were determined using unpaired $t$ tests. Because of missing data we used factorial ANOVA, with time and treatment as nominal variables and Scheffe's posthoc test to correct for multiple comparisons. Changes over time and between groups are indicated by $p$ values for posthoc comparisons within each time period (72-h fast, 8-h glucose drip, 48-h refeed). The effect of periconceptional undernutrition on fetal chest girth increment during late gestation was determined using multiple linear regression (25). Fetal, placental, and birth measurements were analyzed by unpaired $t$ test. All statistical analyses were performed using Statview 5.0.1 (SAS Institute, Cary, NC).

\section{RESULTS}

In cohort 1, 61 ewes entered the study ( $31 \mathrm{UN}, 30 \mathrm{~N})$. Pregnancy rates were not significantly different between groups at scanning $(\mathrm{UN}, 25 / 31,81 \% ; \mathrm{N}, 27 / 30,90 \% ; p=0.3)$. Twenty-eight ewes (18 UN, $10 \mathrm{~N})$ had singleton pregnancies, of whom 5 did not come to surgery due to feeding and health problems, and a further 3 could not be studied due to catheter problems after surgery, leaving $10 \mathrm{UN}$ and $10 \mathrm{~N}$ animals completing the study. In cohort 2, 46 ewes entered the study ( $23 \mathrm{UN}, 23 \mathrm{~N}$ ). Pregnancy rates were lower in the UN ewes, but this did not reach statistical significance (UN, 12/23, 52\%; $\mathrm{N}, 18 / 23,78 \% ; p=0.06)$. Twenty-five ewes $(12 \mathrm{UN}, 13 \mathrm{~N})$ had singleton pregnancies, of whom 4 did not come to surgery due to feeding and health problems, and a further 3 could not be studied due to catheter problems, leaving $10 \mathrm{UN}$ and $8 \mathrm{~N}$ animals completing the study. 
Ewe weights were similar in the two treatment groups $61 \mathrm{~d}$ before mating, but, by the time of mating, UN ewes had lost $\sim 15 \%$ of their body weight and remained lighter than $\mathrm{N}$ ewes at 107 and $131 \mathrm{~d}$ gestation (Fig. 1). There were no differences in ewe weight or weight gain between cohorts, or between UN ewes that subsequently delivered preterm or at term. In cohort 1 at $131 \mathrm{~d}$ fetal weight ( $p=0.2$ ), crown-rump length, limb length, chest girth, and placental size were not significantly different between groups (Table 1). Fetal brain, heart, lung, kidney, and adrenal weights were also not different, but liver weight was $20 \%$ higher in UN fetuses. When organ weights were expressed as a percentage of fetal weight, both liver and heart were larger in UN than in $\mathrm{N}$ fetuses. In cohort 2, fetal girth at surgery was similar in both groups (UN $27 \pm 1 \mathrm{~cm}, \mathrm{~N}$ $28 \pm 1 \mathrm{~cm}$ ). Fetal daily chest girth increment was smaller in $\mathrm{UN}$ fetuses than $\mathrm{N}$ fetuses throughout the period of measurement (Fig. 2). At birth, there were no differences in lamb size (Table 2), but half of the UN group were born prematurely (15), limiting the numbers available for comparison in this group.

Metabolic effects of fasting and refeeding in the $N$ group. Maternal and fetal plasma glucose concentrations fell on fasting, increased on glucose infusion and then fell to baseline levels during refeeding (Fig. 3, Table 3). Maternal and fetal plasma lactate concentrations did not change during fasting or refeeding (Fig. 4, Table 3). Maternal plasma amino nitrogen concentrations fell during fasting and glucose infusion and increased during refeeding, though only the latter reached statistical significance. Fetal plasma amino nitrogen concentrations increased during fasting and did not change during glucose infusion or refeeding (Fig. 5, Table 3). Maternal and fetal plasma urea concentrations increased during fasting, did not change during glucose infusion, and fell during refeeding (Table 3).

Maternal arterial blood $\mathrm{O}_{2}$ content and $\mathrm{PCO}_{2}$ did not change during the study (Fig. 6, Table 4). Fetal $\mathrm{O}_{2}$ content increased

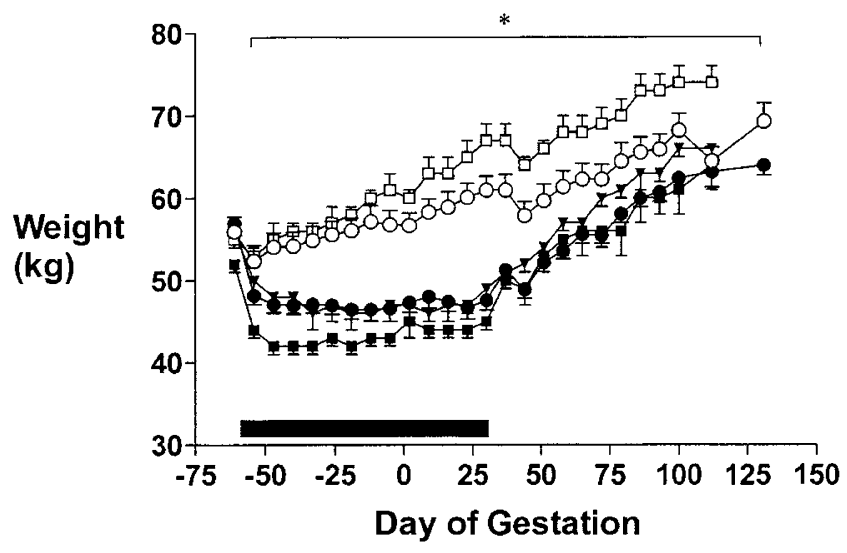

Figure 1. Maternal weight change during study period. Values are mean \pm SEM. Open circles, cohort 1 ewes well fed from $61 \mathrm{~d}$ before to $30 \mathrm{~d}$ after mating (group $\mathrm{N}, n=10$ ). Closed circles, cohort 1 ewes underfed from $61 \mathrm{~d}$ before to $30 \mathrm{~d}$ after mating (group $\mathrm{UN}, n=10$ ). Open squares, cohort 2 ewes well fed from $61 \mathrm{~d}$ before to $30 \mathrm{~d}$ after mating (group $\mathrm{N}$ term, $n=8$ ). Closed squares, cohort 2 ewes underfed from $61 \mathrm{~d}$ before to $30 \mathrm{~d}$ after mating who delivered at term (group UN term, $n=5$ ) Closed triangles, cohort 2 ewes underfed from $61 \mathrm{~d}$ before to $30 \mathrm{~d}$ after mating who delivered preterm (group UN prem, $n=5)$. *Differences between treatment groups $(p<0.0001)$.
Table 1. Fetal and placental size at $131 \mathrm{~d}$ gestation in cohort 1

\begin{tabular}{lcc}
\hline & $\mathrm{N}$ & $\mathrm{UN}$ \\
\hline Fetal weight $(\mathrm{kg})$ & $4.28 \pm 0.21$ & $3.99 \pm 0.08$ \\
Crown-rump length (cm) & $50 \pm 1$ & $50 \pm 0$ \\
Chest girth & $35 \pm 1$ & $35 \pm 0$ \\
Hind leg & $33 \pm 1$ & $33 \pm 0$ \\
Front leg & $29 \pm 0$ & $28 \pm 0$ \\
Brain (g) & $49 \pm 2$ & $50 \pm 1$ \\
Heart (g) & $27 \pm 1$ & $30 \pm 1$ \\
Liver (g) & $109 \pm 7$ & $131 \pm 8^{*}$ \\
Lungs (g) & $114 \pm 4$ & $110 \pm 7$ \\
Kidneys (g) & $24 \pm 1$ & $26 \pm 1$ \\
Adrenals (g) & $0.45 \pm 0.03$ & $0.49 \pm 0.05$ \\
Brain (\%) & $1.2 \pm 0.1$ & $1.3 \pm 0.0$ \\
Heart (\%) & $0.64 \pm 0.02$ & $0.74 \pm 0.02^{*}$ \\
Liver (\%) & $2.6 \pm 0.9$ & $3.3 \pm 0.2^{*}$ \\
Lungs (\%) & $2.7 \pm 0.1$ & $2.8 \pm 0.2$ \\
Kidneys (\%) & $0.57 \pm 0.03$ & $0.65 \pm 0.03$ \\
Adrenals (\%) & $6.011 \pm 0.001$ & $0.012 \pm 0.001$ \\
Maternal uterus (g) & $620 \pm 19$ & $661 \pm 39$ \\
Amniotic fluid (g) & $1052 \pm 155$ & $970 \pm 97$ \\
Placental membranes (g) & $255 \pm 15$ & $289 \pm 21$ \\
Placenta (g) & $411 \pm 29$ & $400 \pm 19$ \\
Placentome number & $79 \pm 7$ & $87 \pm 5$ \\
\hline
\end{tabular}

Values are mean \pm SEM. Percentage figures are organ weight as a proportion of fetal weight. $N$, ewes well fed from $61 \mathrm{~d}$ before to $30 \mathrm{~d}$ after mating $(n=10) ; U N$, ewes underfed from $61 \mathrm{~d}$ before to $30 \mathrm{~d}$ after mating $(n=10)$.

* Significant difference between treatment groups $(p \leq 0.05)$.



Figure 2. Fetal chest girth increment during late gestation in cohort 2. Values are mean \pm SEM. Open circles, ewes well fed from $61 \mathrm{~d}$ before to $30 \mathrm{~d}$ after mating (group $\mathrm{N}, n=8$ ). Closed circles, ewes underfed from $61 \mathrm{~d}$ before to $30 \mathrm{~d}$ after mating, lambs delivered at term (group UN term, $n=5$ ). Closed triangles, ewes underfed from $61 \mathrm{~d}$ before to $30 \mathrm{~d}$ after mating, lambs delivered prematurely (group UN prem, $n=5$ ). *Difference in growth rate between treatment groups $(p<0.0001)$.

on fasting whereas $\mathrm{PCO}_{2}$ decreased. Neither changed during glucose infusion or refeeding. Maternal arterial $\mathrm{pH}$ fell during fasting, did not change on glucose infusion, and increased during refeeding (Fig. 7, Table 4). Fetal arterial pH did not change throughout the study. Maternal and fetal $\mathrm{Hb}$ concentrations did not change during the study.

Metabolic differences between $N$ and $U N$ groups. Plasma glucose concentrations were higher in mothers and fetuses from the UN group during fasting and were not different thereafter (Fig. 3, Table 3). Plasma lactate concentrations were higher in UN fetuses but not mothers throughout fasting, glucose infusion, and refeeding (Fig. 4, Table 3). Furthermore, in $\mathrm{UN}$ but not $\mathrm{N}$ fetuses, plasma lactate concentrations fell 
Table 2. Lamb size at birth in cohort 2

\begin{tabular}{lccc}
\hline & $\mathrm{N}$ & UN term & UN premature \\
\hline Gestation at birth (d) & $145 \pm 1$ & $145 \pm 1$ & $134 \pm 1^{* \dagger}$ \\
Weight $(\mathrm{kg})$ & $5.0 \pm 0.3$ & $4.6 \pm 0.3$ & $3.7 \pm 0.3^{*}$ \\
Crown-rump length $(\mathrm{cm})$ & $51 \pm 1$ & $49 \pm 2$ & $51 \pm 1$ \\
Chest girth (cm) & $42 \pm 1$ & $39 \pm 1$ & $37 \pm 2^{*}$ \\
Hind leg $(\mathrm{cm})$ & $37 \pm 1$ & $36 \pm 1$ & $35 \pm 2$ \\
\hline
\end{tabular}

Values are mean \pm SEM. $N$, ewes well fed from $61 \mathrm{~d}$ before to $30 \mathrm{~d}$ after mating $(n=8) ; U N$, ewes underfed from $61 \mathrm{~d}$ before to $30 \mathrm{~d}$ after mating $(n=$ 5 term and 5 preterm).

* Significantly different from N ( $p \leq 0.05)$.

$\dagger \mathrm{UN}$ prem significantly different from UN term $(p<0.05)$.


Figure 3. Plasma glucose concentrations during maternal fasting, glucose infusion (indicated by dark bar), and refeeding in cohort 1 . Values are mean \pm SEM. Open circles, ewes well fed from $61 \mathrm{~d}$ before to $30 \mathrm{~d}$ after mating (group $\mathrm{N}, n=10$ ). Closed circles, ewes underfed from $61 \mathrm{~d}$ before to $30 \mathrm{~d}$ after mating, lambs delivered at term (group UN, $n=10$ ). *Difference between treatment groups $(p<0.05)$. **Difference between treatment groups $(p<$ $0.01)$.

Table 3. Plasma metabolite concentrations during maternal fasting and refeeding in cohort 1

\begin{tabular}{|c|c|c|c|c|}
\hline & \multicolumn{2}{|c|}{ Maternal } & \multicolumn{2}{|c|}{ Fetal } \\
\hline & $\mathrm{N}$ & UN & $\mathrm{N}$ & UN \\
\hline \multicolumn{5}{|l|}{ Glucose $(\mathrm{mM})$} \\
\hline Baseline & $3.0 \pm 0.1$ & $3.1 \pm 0.1$ & $0.8 \pm 0.1$ & $0.9 \pm 0.1$ \\
\hline 72-h fasted & $1.5 \pm 0.1 \dagger$ & $1.8 \pm 0.2 \dagger \S$ & $0.4 \pm 0.0 \dagger$ & $0.5 \pm 0.0 \dagger \S$ \\
\hline 8-h glucose drip & $3.5 \pm 0.0 \dagger$ & $3.6 \pm 0.2 \dagger$ & $0.9 \pm 0.1 \dagger$ & $1.0 \pm 0.1 \dagger$ \\
\hline 48-h refed & $3.0 \pm 0.1 \dagger$ & $3.1 \pm 0.1$ & $0.8 \pm 0.0 \dagger$ & $0.8 \pm 0.1 \dagger$ \\
\hline \multicolumn{5}{|l|}{ Lactate (mM) } \\
\hline Baseline & $0.5 \pm 0.1$ & $0.5 \pm 0.0$ & $1.6 \pm 0.2$ & $1.9 \pm 0.1$ \\
\hline 72-h fasted & $0.6 \pm 0.1$ & $0.6 \pm 0.0$ & $1.3 \pm 0.1$ & $1.6 \pm 0.0 \dagger \S$ \\
\hline 8-h glucose drip & $0.5 \pm 0.1$ & $0.6 \pm 0.1$ & $1.6 \pm 0.1$ & $2.0 \pm 0.1 \dagger \S$ \\
\hline 48-h refed & $0.4 \pm 0.0$ & $0.4 \pm 0.1$ & $1.7 \pm 0.1$ & $2.4 \pm 0.2 \S$ \\
\hline \multicolumn{5}{|c|}{ Amino nitrogen $(\mathrm{mM})$} \\
\hline Baseline & $3.5 \pm 0.2$ & $3.7 \pm 0.3^{*}$ & $5.2 \pm 0.2$ & $3.7 \pm 0.3^{*}$ \\
\hline 72-h fasted & $2.4 \pm 0.1$ & $3.0 \pm 0.3 \S$ & $6.2 \pm 0.2 \dagger$ & $3.0 \pm 0.3 \S$ \\
\hline 8-h glucose drip & $2.3 \pm 0.1$ & $2.6 \pm 0.2$ & $5.6 \pm 0.2$ & $2.6 \pm 0.2 \S$ \\
\hline 48-h refed & $3.6 \pm 0.1 \dagger$ & $3.5 \pm 0.2$ & $6.0 \pm 0.2$ & $3.5 \pm 0.2$ \\
\hline \multicolumn{5}{|l|}{ Urea $(\mathrm{mM})$} \\
\hline Baseline & $5.8 \pm 0.3$ & $5.8 \pm 0.5$ & $6.5 \pm 0.3$ & $6.5 \pm 0.4$ \\
\hline 72-h fasted & $8.3 \pm 1.0$ & $7.9 \pm 0.6 \dagger$ & $9.2 \pm 0.7 \dagger$ & $8.7 \pm 0.6 \dagger$ \\
\hline 8-h glucose drip & $6.1 \pm 0.6$ & $6.7 \pm 0.6$ & $7.7 \pm 0.7$ & $7.8 \pm 0.5$ \\
\hline 48-h refed & $3.1 \pm 0.3 \dagger$ & $3.8 \pm 0.5 \dagger$ & $3.8 \pm 0.2 \dagger$ & $4.0 \pm 0.2 \dagger$ \\
\hline
\end{tabular}

Values are mean \pm SEM. $N$, ewes well fed from $61 \mathrm{~d}$ before to $30 \mathrm{~d}$ after mating $(n=10)$; UN, ewes underfed from $61 \mathrm{~d}$ before to $30 \mathrm{~d}$ after mating $(n=10)$.

* Difference between treatment groups at baseline $(p<0.05)$.

$\dagger$ Change over time within this time period $(p<0.05)$.

$\S$ Difference between treatment groups within this period $(p<0.05)$.

during fasting and increased during glucose infusion. Plasma amino nitrogen concentrations were higher in mothers and lower in fetuses in the UN group at baseline and during fasting but were not different between treatment groups during refeed-
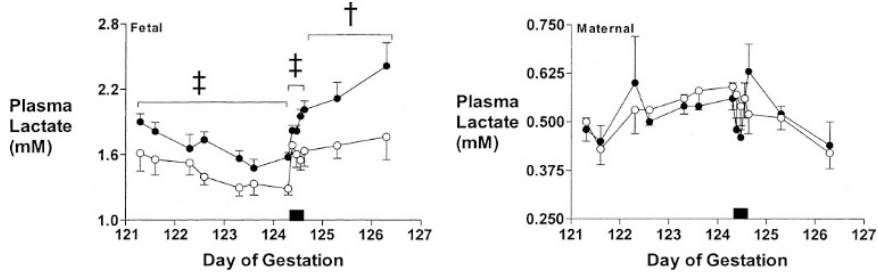

Figure 4. Plasma lactate concentrations during maternal fasting, glucose infusion (indicated by dark bar), and refeeding in cohort 1 . Values are mean \pm SEM. Open circles, ewes well fed from $61 \mathrm{~d}$ before to $30 \mathrm{~d}$ after mating (group $\mathrm{N}, n=10$ ). Closed circles, ewes underfed from $61 \mathrm{~d}$ before to $30 \mathrm{~d}$ after mating (group $\mathrm{UN}, n=10$ ). $\dagger$ Difference between treatment groups $(p<$ $0.001)$. $\ddagger$ Difference between treatment groups $(p<0.0001)$.
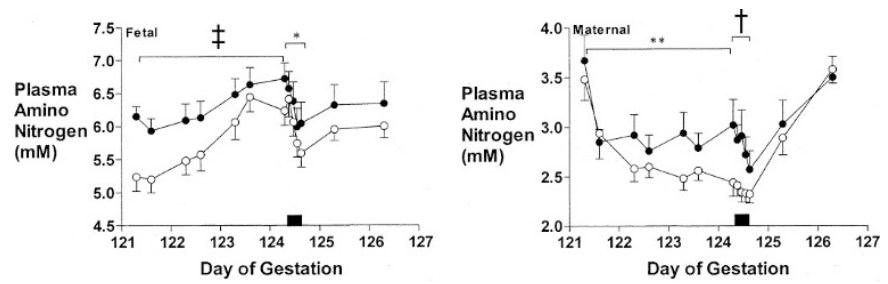

Figure 5. Plasma amino nitrogen concentrations during maternal fasting, glucose infusion (indicated by dark bar), and refeeding in cohort 1 . Values are mean \pm SEM. Open circles, ewes well fed from $61 \mathrm{~d}$ before to $30 \mathrm{~d}$ after mating (group N, $n=10)$. Closed circles, ewes underfed from $61 \mathrm{~d}$ before to $30 \mathrm{~d}$ after mating (group UN, $n=10$ ). *Difference between treatment groups $(p<0.05)$. **Difference between treatment groups $(p<0.01)$. †Difference between treatment groups $(p<0.001)$. Difference between treatment groups $(p<0.0001)$.
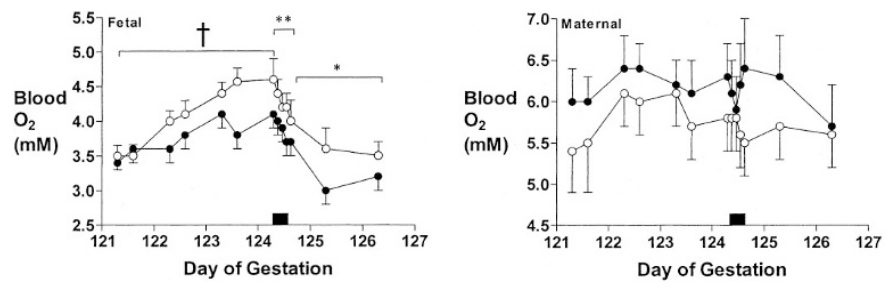

Figure 6. Arterial blood oxygen content during maternal fasting, glucose infusion (indicated by dark bar), and refeeding in cohort 1 . Values are mean \pm SEM. Open circles, ewes well fed from $61 \mathrm{~d}$ before to $30 \mathrm{~d}$ after mating (group N, $n=10$ ). Closed circles, ewes underfed from $61 \mathrm{~d}$ before to $30 \mathrm{~d}$ after mating (group UN, $n=10$ ). *Difference between treatment groups $(p<0.05)$. **Difference between treatment groups $(p<0.01)$. †Difference between treatment groups $(p<0.001)$.

ing (Fig. 5, Table 3). Plasma urea concentrations were not different between treatment groups at any stage of the study (Table 3).

Fetal but not maternal arterial blood $\mathrm{O}_{2}$ content was lower in the UN group throughout the study, whereas $\mathrm{PCO}_{2}$ was higher. Fetal $\mathrm{O}_{2}$ content did not increase in UN fetuses during fasting, but did fall during refeeding. Fetal but not maternal arterial $\mathrm{pH}$ was lower in UN group throughout the study. Maternal but not fetal $\mathrm{Hb}$ concentrations were higher in the UN group throughout the study, but this reached statistical significance only during fasting and glucose infusion.

Endocrine effects of fasting and refeeding in the $N$ group. Maternal and fetal plasma insulin concentrations fell during fasting, increased during glucose infusion, then increased in mothers but fell in fetuses during refeeding (Table 5). Maternal and fetal plasma IGF-I concentrations fell during fasting, did 
Table 4. Arterial blood gases and hemoglobin during maternal fasting and refeeding in cohort 1

\begin{tabular}{|c|c|c|c|c|}
\hline & \multicolumn{2}{|c|}{ Maternal } & \multicolumn{2}{|c|}{ Fetal } \\
\hline & $\mathrm{N}$ & UN & $\mathrm{N}$ & UN \\
\hline \multicolumn{5}{|l|}{$\mathrm{Po}_{2}(\mathrm{~mm} \mathrm{Hg})$} \\
\hline Baseline & $129 \pm 5$ & $126 \pm 4$ & $19 \pm 1$ & $19 \pm 1$ \\
\hline 72-h fasted & $126 \pm 5$ & $111 \pm 3$ & $23 \pm 1 \dagger$ & $21 \pm 1 \S$ \\
\hline 8-h glucose drip & $117 \pm 4$ & $115 \pm 6 \S$ & $20 \pm 1$ & $19 \pm 1$ \\
\hline 48-h refed & $125 \pm 5$ & $119 \pm 4 \S$ & $19 \pm 1$ & $18 \pm 1$ \\
\hline \multicolumn{5}{|l|}{$\mathrm{PCO}_{2}(\mathrm{~mm} \mathrm{Hg})$} \\
\hline Baseline & $31 \pm 1$ & $32 \pm 1$ & $50 \pm 1$ & $51 \pm 1$ \\
\hline 72-h fasted & $32 \pm 1$ & $33 \pm 0$ & $46 \pm 1 \dagger$ & $48 \pm 1 \dagger \S$ \\
\hline 8-h glucose drip & $31 \pm 1$ & $31 \pm 1$ & $46 \pm 1$ & $49 \pm 2 \S$ \\
\hline $48-\mathrm{h}$ refed & $35 \pm 1$ & $34 \pm 1$ & $51 \pm 1$ & $53 \pm 2 \S$ \\
\hline \multicolumn{5}{|l|}{$\mathrm{O}_{2}(\mathrm{mM})$} \\
\hline Baseline & $5.4 \pm 0.5$ & $6.0 \pm 0.4$ & $3.5 \pm 0.2$ & $3.4 \pm 0.1$ \\
\hline 72-h fasted & $5.8 \pm 0.4$ & $6.3 \pm 0.4$ & $4.6 \pm 0.3 \dagger$ & $4.1 \pm 0.2 \S$ \\
\hline 8-h glucose drip & $5.5 \pm 0.4$ & $6.4 \pm 0.6$ & $4.0 \pm 0.3$ & $3.7 \pm 0.2 \S$ \\
\hline 48-h refed & $5.6 \pm 0.5$ & $5.7 \pm 0.5$ & $3.5 \pm 0.2$ & $3.2 \pm 0.2 \dagger \S$ \\
\hline \multicolumn{5}{|l|}{$\mathrm{pH}$} \\
\hline Baseline & $7.49 \pm 0.01$ & $7.48 \pm 0.02$ & $7.38 \pm 0.01$ & $7.36 \pm 0.01$ \\
\hline 72-h fasted & $7.43 \pm 0.01 \dagger$ & $7.44 \pm 0.01 \dagger$ & $7.39 \pm 0.01$ & $7.38 \pm 0.00 \S$ \\
\hline 8-h glucose drip & $7.43 \pm 0.01$ & $7.44 \pm 0.01$ & $7.38 \pm 0.01$ & $7.37 \pm 0.00 \S$ \\
\hline 48-h refed & $7.50 \pm 0.01 \dagger$ & $7.49 \pm 0.01 \dagger$ & $7.40 \pm 0.0$ & $7.38 \pm 0.01$ \\
\hline \multicolumn{5}{|l|}{$\mathrm{Hb}(\mathrm{g} \%)$} \\
\hline Baseline & $8.6 \pm 0.8$ & $9.6 \pm 0.6$ & $10.5 \pm 0.3$ & $10.7 \pm 0.4$ \\
\hline 72-h fasted & $9.3 \pm 0.7$ & $10.2 \pm 0.7 \S$ & $11.8 \pm 0.6$ & $11.4 \pm 0.4$ \\
\hline 8-h glucose drip & $8.8 \pm 0.6$ & $10.4 \pm 0.9 \S$ & $11.5 \pm 0.5$ & $11.1 \pm 0.4$ \\
\hline 48-h refed & $9.0 \pm 0.7$ & $9.2 \pm 0.8$ & $11.3 \pm 0.7$ & $10.9 \pm 0.4$ \\
\hline
\end{tabular}

Values are mean \pm SEM. $N$, ewes well fed from $61 \mathrm{~d}$ before to $30 \mathrm{~d}$ after mating $(n=10)$; $U N$, ewes underfed from $61 \mathrm{~d}$ before to $30 \mathrm{~d}$ after mating $(n=10)$

$\dagger$ Change over time within this time period $(p<0.05)$.

$\S$ Difference between treatment groups within this period $(p<0.05)$.
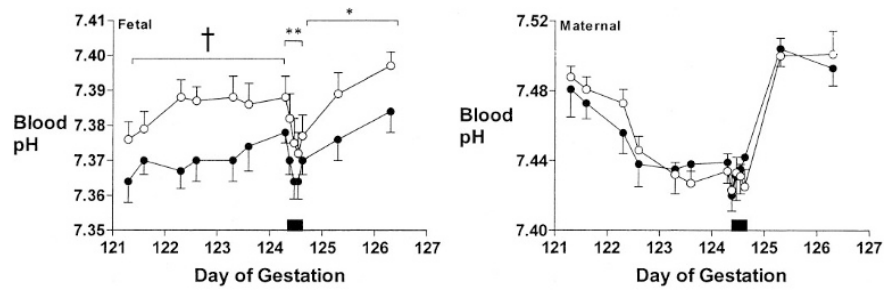

Figure 7. Arterial blood $\mathrm{pH}$ during maternal fasting, glucose infusion (indicated by dark bar), and refeeding in cohort 1 . Values are mean \pm SEM. Open circles, ewes well fed from $61 \mathrm{~d}$ before to $30 \mathrm{~d}$ after mating (group $\mathrm{N}, n=10$ ). Closed circles, ewes underfed from $61 \mathrm{~d}$ before to $30 \mathrm{~d}$ after mating (group $\mathrm{UN}, n=10)$. *Difference between treatment groups $(p<0.05)$.**Difference between treatment groups $(p<0.01)$. $\dagger$ Difference between treatment groups $(p$ $<0.0001)$.

not change during glucose infusion, but increased on refeeding. Maternal and fetal plasma IGFBP-1 concentrations increased during fasting, fell during glucose infusion, and did not change on refeeding. Fetal but not maternal plasma oPL concentrations increased on fasting then did not change on glucose infusion or refeeding.

Endocrine differences between $N$ and UN groups. Plasma insulin levels were similar in the two groups at baseline. Maternal plasma insulin concentrations were higher in UN than $\mathrm{N}$ groups throughout the study. Fetal plasma insulin concentrations were higher in UN fetuses only during fasting. Maternal but not fetal plasma IGF-I concentrations were slightly lower in $\mathrm{UN}$ than $\mathrm{N}$ groups throughout the study, but this
Table 5. Plasma hormone concentrations during maternal fasting and refeeding in cohort 1

\begin{tabular}{lccccc}
\hline & \multicolumn{2}{c}{ Maternal } & & \multicolumn{2}{c}{ Fetal } \\
\cline { 2 - 3 } \cline { 5 - 6 } \cline { 5 - 6 } & $\mathrm{N}$ & $\mathrm{UN}$ & & $\mathrm{N}$ & $\mathrm{UN}$ \\
\hline Insulin (pM) & & & & \\
$\quad$ Baseline & $81 \pm 12$ & $63 \pm 13$ & & $34 \pm 4$ & $40 \pm 6$ \\
72-h fasted & $3.7 \pm 0.4 \dagger$ & $6.8 \pm 2.1 \dagger$ & & $11 \pm 2 \dagger$ & $12 \pm 3 \dagger \S$ \\
8-h glucose drip & $21 \pm 7$ & $49 \pm 11 \dagger \S$ & $72 \pm 7 \dagger$ & $71 \pm 8 \dagger$ \\
48-h refed & $71 \pm 9 \dagger$ & $99 \pm 25 \S$ & $39 \pm 5 \dagger$ & $31 \pm 6 \dagger$ \\
IGF-1 (nM) & & & & \\
Baseline & $20 \pm 1$ & $18 \pm 1$ & & $12 \pm 0$ & $13 \pm 1$ \\
72-h fasted & $9.9 \pm 0.9 \dagger$ & $7.8 \pm 1.1 \dagger \S$ & $8.3 \pm 0.5 \dagger$ & $8.6 \pm 1.1 \dagger$ \\
8-h glucose drip & $11 \pm 1$ & $9.1 \pm 1.1$ & & $9.2 \pm 0.5$ & $9.3 \pm 1.0$ \\
48-h refed & $16 \pm 1 \dagger$ & $14 \pm 1$ & & $12 \pm 1 \dagger$ & $11 \pm 1$ \\
IGFBP-1 (nM) & & & & \\
Baseline & $0.4 \pm 0.1$ & $0.9 \pm 0.2$ & & $3.2 \pm 0.9$ & $3.8 \pm 0.9$ \\
72-h fasted & $9.7 \pm 0.8 \dagger$ & $9.9 \pm 1.3 \dagger$ & & $15 \pm 2 \dagger$ & $16 \pm 2 \dagger$ \\
8-h glucose drip & $2.8 \pm 0.4 \dagger$ & $2.4 \pm 0.4 \dagger$ & & $2.6 \pm 0.4 \dagger$ & $3.7 \pm 0.4 \dagger$ \\
48-h refed & $0.7 \pm 0.2 \dagger$ & $0.9 \pm 0.4$ & & $3.0 \pm 0.5$ & $6.1 \pm 1.8$ \\
PL (nM) & & & & \\
Baseline & $3.5 \pm 0.4$ & $5.0 \pm 0.5 *$ & & $0.41 \pm 0.02$ & $0.50 \pm 0.02^{*}$ \\
72-h fasted & $5.6 \pm 0.5$ & $7.1 \pm 0.7 \S$ & & $0.55 \pm 0.03$ & $0.59 \pm 0.03 \S$ \\
8-h glucose drip & $5.3 \pm 0.4$ & $6.3 \pm 0.5 \S$ & & $0.50 \pm 0.03$ & $0.58 \pm 0.03 \S$ \\
48-h refed & $4.9 \pm 0.4$ & $5.8 \pm 0.5 \S$ & $0.53 \pm 0.05$ & $0.54 \pm 0.03$ \\
\hline
\end{tabular}

Values are mean \pm SEM. $N$, ewes well fed from $61 \mathrm{~d}$ before to $30 \mathrm{~d}$ after mating $(n=10)$; $U N$, ewes underfed from $61 \mathrm{~d}$ before to $30 \mathrm{~d}$ after mating $(n=10)$.

* Difference between treatment groups at baseline $(p<0.05)$.

$\dagger$ Change over time within this time period $(p<0.05)$.

$\S$ Difference between treatment groups within this period $(p<0.05)$.

different reached statistical significance only during fasting. Maternal and fetal plasma IGFBP-1 concentrations were not different between treatment groups at any stage of the study. Maternal and fetal plasma oPL concentrations were higher in $\mathrm{UN}$ than $\mathrm{N}$ groups throughout the study.

\section{DISCUSSION}

Although the association between small size at birth and later disease risk is now widely accepted, the mechanisms underlying these phenomena remain uncertain. Few studies have addressed the effects of intrauterine undernutrition independently of size at birth, and few have distinguished the direct effects of undernutrition on the fetus, separately from possible downstream postnatal effects as a result of that undernutrition. In this study, we have shown that undernutrition in the period around conception in the sheep alters fetal growth trajectory throughout gestation right until delivery, although it has no significant effect on size at birth. Furthermore, we have shown that maternal undernutrition until d 30 of gestation alters maternal and fetal metabolic and endocrine responses to a brief nutritional insult in late gestation in a way that appears to reflect fetoplacental adaptations to maintain ongoing slow fetal growth and survival in the face of such an insult. These findings may have important implications for the likely impact of early pregnancy events on lifelong health.

We have previously demonstrated that fetuses whose mothers were undernourished in the periconceptional period grow more slowly in late gestation than those whose mothers were well nourished (19). Unfortunately, for logistical reasons we 
were unable to measure growth in the same way in the cohort 1 fetuses reported in the current study. However, in the concurrent cohort 2 fetuses subject to the same nutritional manipulations we also found reduced growth rate very similar to that observed in earlier studies, but in this case persisting throughout late gestation until delivery. Slow fetal growth would be consistent with reduced fetal consumption of metabolic substrates, and hence with the increased fetal plasma concentrations of glucose and lactate that we observed in UN fetuses.

Furthermore, in our previous studies we showed that fetal growth responses to severe maternal undernutrition in late gestation are also influenced by maternal undernutrition during the periconceptional period (19). Fetuses of well-nourished ewes slow their growth promptly in response to late-gestation undernutrition, whereas fetuses whose mothers were undernourished in the periconceptional period do not show the same slowing of growth in response to late maternal undernutrition. In the current study, maternal fasting resulted in a rise in fetal plasma concentrations of amino nitrogen and oxygen and a fall in $\mathrm{PCO}_{2}$ in $\mathrm{N}$ but not $\mathrm{UN}$ fetuses. These changes are similar to those observed previously in response to maternal undernutrition (33) and would be consistent with slowed growth and hence reduced oxidative metabolic demand only in $\mathrm{N}$ fetuses.

Interestingly, this slow fetal growth trajectory was not reflected in overall fetal size. Fetal and placental weights were not different between groups at $131 \mathrm{~d}$ gestation or at delivery. However, liver, heart, and kidney size was relatively larger in UN fetuses at $131 \mathrm{~d}$. Similar findings have been reported previously after periconceptional maternal undernutrition (34). However, the mechanisms by which such changes are induced and their significance, if any, for long-term health and disease risk remain obscure. Thinness at birth has been positively correlated with impaired glucose tolerance in later life (35-37), suggesting that the reduced girth increment we observed in this study may be related to the alteration in pancreatic function reported previously (16).

Compared with $\mathrm{N}$ fetuses, $\mathrm{UN}$ fetuses had reduced blood oxygen content, increased $\mathrm{PCO}_{2}$, and reduced $\mathrm{pH}$ throughout the study. Although all these changes were small, together they may suggest limitation of placental function for gas exchange relative to oxidative metabolic demands in UN fetuses. The increased fetal blood lactate concentrations in UN fetuses may also reflect altered placental function, as a large proportion of circulating lactate is of placental origin (38), and placental lactate production may be preserved in the face of reduced glucose supply $(33,38)$. Because lactate is not taken back up by the sheep placenta from the fetal circulation, the net result may be to conserve carbohydrate in the fetal circulation. Maternal undernutrition throughout pregnancy in guinea pigs results in reduced placental surface area for gas exchange in late gestation and in increased diffusion distance (39). Our data suggest that altered placental function may also be induced by maternal undernutrition ending at $30 \mathrm{~d}$ gestation, before development of the cotyledonary placenta in sheep. Potential signals that may mediate such effects remain uncertain.

In the UN ewes, glucose, amino nitrogen, insulin, and PL concentrations were all higher than in N ewes. PL contributes to insulin resistance (40) and substrate release in the maternal circulation (41). In rats, maternal undernutrition during pregnancy also results in impaired glucose tolerance in the dam (42). Together these findings suggest that UN ewes had a greater degree of insulin resistance, which should make circulating substrates more freely available to the fetus. This may represent a means by which undernutrition in early pregnancy results in maternal metabolic and endocrine adaptations that help maintain nutrient supply to the fetus and hence maintain continued slow fetal growth in the face of potential ongoing nutritional limitation during late gestation.

However, UN fetuses also had increased PL concentrations, and concentrations of insulin and IGF-I similar to those of N fetuses. Inasmuch as these anabolic hormones, together with apparently adequate substrate supply, would be expected to lead to increased fetal growth, these findings together suggest either that UN fetuses have a degree of resistance to these hormones, or alternatively that the regulation of fetal growth is altered in some other way in UN fetuses. We and others have previously found evidence of resistance to the metabolic effects of IGF-1 and insulin in fetuses whose growth is limited by placental embolization (43) or uterine artery ligation (44). The mechanisms of such resistance are unknown, although it may be relevant that early activation of the fetal HPA axis was observed in similar fetuses (45), and elevated fetal plasma cortisol levels could contribute both to slowed fetal growth (46) and also to relative insulin and IGF-I resistance.

PL has been shown to stimulate glycogen synthesis (47) and inhibit glycogen breakdown in the fetal compartment (48). Higher plasma PL concentrations in UN fetuses may therefore also represent a further mechanism by which fetal carbohydrates stores may be conserved in the face of potentially limited supply. In a previous study, we reported that during maternal fasting plasma PL concentrations were higher in fetuses whose mothers had experienced poor nutrition before the study (20). In that study it was impossible for us to clarify when the onset of poor maternal nutrition occurred. However, the current study suggests that the periconceptional period may be very important.

The observed fall in maternal and fetal plasma concentrations of IGF-I after maternal fasting for $72 \mathrm{~h}$ and the slow return to baseline levels on refeeding was consistent with our previous studies (49). Similarly, the increase in maternal and fetal plasma concentrations of IGFBP-1 on maternal fasting and the rapid fall on maternal glucose infusion and refeeding are in agreement with our previous findings (50). IGFBP-1 recovered more quickly than IGF-I on refeeding, and this finding is consistent with the acute regulation of circulating IGFBP-1 by glucose (51). Previously, we have reported that both maternal and fetal plasma IGFBP-3 levels fall on maternal fasting and rise slowly on refeeding (50). Although we did not measure IGFBP-3 levels in this study, it is very likely because of the large binding capacity of IGFBP-3 that a slow increase on refeeding would impact on the recovery of plasma IGF-1 concentrations. In this study we observed no effect of periconceptional undernutrition on fetal plasma IGF-I and IGFBP-1 baseline values or responses to maternal fasting. The lack of any evidence of intrauterine growth restriction in fetuses from 
the periconceptionally undernourished group is consistent with the lack of difference in IGF-I and IGFBP-1 values.

Maternal undernutrition in early to mid-pregnancy has been reported to affect the HPA axis and cardiovascular function in the late-gestation fetal sheep $(17,18)$ and postnatal lamb (52), with little effect on fetal or birth size. Studies of the offspring of Dutch famine survivors also suggest that maternal starvation in the periconceptional period increases the incidence of cardiovascular disease during adult life with little effect on weight at birth $(10,11)$. These findings raise the interesting possibility that maternal nutrition around the time of conception and/or in very early pregnancy alters the physiology of the developing fetus in a way that leads to increased disease risk in later life independent of size at birth. However, to date there have been few attempts to address this possibility experimentally. Furthermore, there is little information as to whether such altered physiology develops in utero, or only in response to the postnatal environment.

We have previously shown that periconceptional maternal undernutrition similar to that described in this study resulted in altered fetal growth (19), pancreatic beta cell function (16), and regulation of the HPA axis (45) and IGF axis in late gestation (14). This study augments those earlier studies in demonstrating that fetal growth is altered throughout late gestation until delivery, and that both maternal and fetal metabolic and endocrine status are perturbed in late gestation after periconceptional undernutrition. These changes might be expected to conserve fetal substrate supplies in late gestation. Such adaptations may allow continued fetal growth and survival in the face of potential ongoing nutrient limitation. The nature of the putative signals in early pregnancy that determine these adaptations deserves further investigation.

Acknowledgments. The authors thank Geoff Hobson, Samantha Rossenrode, Sarah Sargison, Andrzej Surus, Toni Smith-Wong, and Pierre Van Zijl for technical support.

\section{REFERENCES}

1. Hales CN, Barker DJ, Clark PM, Cox LJ, Fall C, Osmond C, Winter PD 1991 Fetal and infant growth and impaired glucose tolerance at age 64. BMJ 303:1019-1022

2. Seckl JR, Benediktsson R, Lindsay RS, Brown RW 1995 Placental 11 betahydroxysteroid dehydrogenase and the programming of hypertension. J Steroid Biochem Mol Biol 55:447-455

3. Phillips ID, Simonetta G, Owens JA, Robinson JS, Clarke IJ, McMillen IC 1996 Placental restriction alters the functional development of the pituitary-adrenal axis in the sheep fetus during late gestation. Pediatr Res 40:861-866

4. Jansson T, Lambert GW 1999 Effect of intrauterine growth restriction on blood pressure, glucose tolerance and sympathetic nervous system activity in the rat at 3-4 months of age. J Hypertens 17:1239-1248

5. Langley-Evans S, Jackson A 1996 Intrauterine programming of hypertension: nutrient-hormone interactions. Nutr Rev 54:163-169

6. Sohlstrom A, Katsman A, Kind KL, Roberts CT, Owens PC, Robinson JS, Owens JA 1998 Food restriction alters pregnancy-associated changes in IGF and IGFBP in the guinea pig. Am J Physiol 274:E410-E416

7. Kind KL, Clifton PM, Katsman AI, Tsiounis M, Robinson JS, Owens JA 1999 Restricted fetal growth and the response to dietary cholesterol in the guinea pig. Am J Physiol 277:R1675-R1682

8. Harding JE, Johnston BM 1995 Nutrition and fetal growth. Reprod Fertil Dev 7:539-547

9. Vickers MH, Ikenasio BA, Breier BH 2001 IGF-1 treatment reduces hyperphagia, obesity, and hypertension in metabolic disorders induced by fetal programming. Endocrinology 142:3964-3973

10. Ravelli AC, van der Meulen JH, Michels RP, Osmond C, Barker DJ, Hales CN Bleker OP 1998 Glucose tolerance in adults after prenatal exposure to famine. Lancet 351:173-177
11. Roseboom TJ, van der Meulen JH, Osmond C, Barker DJ, Ravelli AC, SchroederTanka JM, van Montfrans GA, Michels RP, Bleker OP 2000 Coronary heart disease after prenatal exposure to the Dutch famine, 1944-45. Heart 84:595-598

12. Kwong WY, Wild AE, Roberts P, Willis AC, Fleming TP 2000 Maternal undernutrition during the preimplantation period of rat development causes blastocyst abnormalities and programming of postnatal hypertension. Development 127:4195-4202

13. Barnes FL 2000 The effects of the early uterine environment on the subsequent development of embryo and fetus. Theriogenology 53:649-658

14. Gallaher BW, Breier BH, Keven CL, Harding JE, Gluckman PD 1998 Fetal programming of insulin-like growth factor (IGF)-I and IGF-binding protein-3: evidence for an altered response to undernutrition in late gestation following exposure to periconceptual undernutrition in the sheep. J Endocrinol 159:501-508

15. Bloomfield FH, Oliver MH, Hawkins P, Campbell M, Phillips DJ, Breier BH, Gluckman PD, Challis JR, Harding JE 2003 A periconceptual nutritional origin for non-infectious preterm birth. Science 300:606

16. Oliver MH, Hawkins P, Breier BH, van Zijl PL, Sargison SA, Harding JE 2001 Maternal undernutrition during the periconceptual period increases plasma taurine levels and insulin response to glucose but not arginine in the late gestational fetal sheep. Endocrinology 142:4576-4579

17. Hawkins P, Steyn C, McGarrigle HH, Saito T, Ozaki T, Stratford LL, Noakes DE, Hanson MA 1999 Effect of maternal nutrient restriction in early gestation on development of the hypothalamic-pituitary-adrenal axis in fetal sheep at $0.8-0.9$ of gestation. J Endocrinol 163:553-561

18. Hawkins P, Steyn C, Ozaki T, Saito T, Noakes DE, Hanson MA 2000 Effect of maternal undernutrition in early gestation on ovine fetal blood pressure and cardiovascular reflexes. Am J Physiol Regul Integr Comp Physiol 279:R340-R348

19. Harding JE 1997 Periconceptional nutrition determines the fetal growth response to actue maternal undernutrition in fetal sheep of late gestation. Prenat Neonat Med 2:310-319

20. Oliver MH, Harding JE, Breier BH, Evans PC, Gluckman PD 1992 The nutritional regulation of circulating placental lactogen in fetal sheep. Pediatr Res 31:520-523

21. Oliver MH, Harding JE, Breier BH, Gluckman PD 1996 Fetal insulin-like growth factor (IGF)-I and IGF-II are regulated differently by glucose or insulin in the sheep fetus. Reprod Fertil Dev 8:167-172

22. Mellor DJ 1987 Feeding pregnant ewes and newborn lambs during experiment. In: Nathanielsz PW (ed) Animal Models in Fetal Medicine. VI. Perinatology Press, Ithaca, NY, pp 55-92

23. Wheaton JE, Carlson KM, Windels HF, Johnston LJ 1993 CIDR: A new progesterone-releasing intravaginal device for induction of estrus and cycle control in sheep and goats. Anim Reprod Sci 33:127-141

24. Mellor DJ, Murray L 1982 Effects on the rate of increase in fetal girth of refeeding ewes after short periods of severe undernutrition during late pregnancy. Res Vet Sci 32:377-382

25. Harding JE 1997 Prior growth rate determines the fetal growth response to acute maternal undernutrition in fetal sheep of late gestation. Prenat Neonat Med 2:300-309

26. Kunst A, Draeger B, Ziegenhorn J 1984 Colorimetric methods with glucose oxidase and peroxidase. In: Bergmeyer HU (ed) Methods of Enzymatic Analysis. Verlag Chemie, Weinheim, Germany, pp 178-185

27. Kerscher L, Ziegenhorn J 1985 Urea. In: Bergmeyer HU (ed) Methods of Enzymatic Analysis. Verlag Chemie, Weinheim, Germany, pp 444-453

28. Ashour MB, Gee SJ, Hammock BD 1987 Use of a 96-well microplate reader for measuring routine enzyme activities. Anal Biochem 166:353-360

29. Evans PC, Ffolliott Powell FM, Harding JE 1993 A colorimetric assay for amino nitrogen in small volumes of blood: reaction with beta-naphthoquinone sulfonate. Anal Biochem 208:334-337

30. Oliver MH, Harding JE, Breier BH, Evans PC, Gluckman PD 1993 Glucose but not a mixed amino acid infusion regulates plasma insulin-like growth factor-I concentrations in fetal sheep. Pediatr Res 34:62-65

31. Blum WF, Breier BH 1994 Radioimmunoassays for IGFs and IGFBPs. Growth Regul 4:11-19

32. Vickers MH, Casey PJ, Champion ZJ, Gravance CG, Breier BH 1999 IGF-I treatment increases motility and improves morphology of immature spermatozoa in the GHdeficient dwarf (dw/dw) rat. Growth Horm IGF Res 9:236-240

33. Harding JE, Gluckman PD 2001 Growth, metabolic and endocrine adaptations to fetal undernutrition. In: Barker JDP (ed) Fetal Origins of Cardiovascular Disease and Lung Disease. Marcel Dekker, New York, pp 181-197

34. Harding JE 2001 The nutritional basis of the fetal origins of adult disease. Int $\mathrm{J}$ Epidemiology 30:15-23

35. Phipps K, Barker DJ, Hales CN, Fall CH, Osmond C, Clark PM 1993 Fetal growth and impaired glucose tolerance in men and women. Diabetologia 36:225-228

36. Lithell HO, McKeigue PM, Berglund L, Mohsen R, Lithell UB, Leon DA 1996 Relation of size at birth to non-insulin dependent diabetes and insulin concentrations in men aged 50-60 years. BMJ 312:406-410

37. Eriksson JG, Forsen T, Tuomilehto J, Jaddoe VW, Osmond C, Barker DJ 2002 Effects of size at birth and childhood growth on the insulin resistance syndrome in elderly individuals. Diabetalogia 45:342-348

38. Battaglia FC, Meschia G 1978 Principal substrates of fetal metabolism. Physiol Rev 58:499-527

39. Roberts CT, Sohlstrom A, Kind KL, Earl RA, Khong TY, Robinson JS, Owens PC, Owens JA 2001 Maternal food restriction reduces the exchange surface area and increases the barrier thickness of the placenta in the guinea-pig. Placenta 22:177-185

40. Ryan EA, Enns L 1988 Role of gestational hormones in the induction of insulin resistance. J Clin Endocrinol Metab 67:341-347

41. Thordarson G, McDowell GH, Smith SV, Ileys S, Forsyth IA 1987 Effects of continuous intravenous infusion of an ovine placental extract enriched in placental 
lactogen on plasma hormones, metabolites and metabolite biokinetics. J Endocrinol 113:277-283

42. Alvarez C, Martin MA, Goya L, Bertin E, Portha B, Pascual-Leone AM 1997 Contrasted impact of maternal rat food restriction on the fetal endocrine pancreas. Endocrinology 138:2267-2273

43. Jensen EC, Harding JE, Bauer MK, Gluckman PD 1999 Metabolic effects of IGF-I in the growth retarded fetal sheep. J Endocrinol 161:485-494

44. Frampton RJ, Jonas HA, MacMahon RA, Larkins RG 1990 Failure of IGF-1 to affect protein turnover in muscle from growth-retarded neonatal rats. J Dev Physiol 13:125-133

45. Bloomfield FH, Oliver MH, Hawkins P, Holloway AC, Campbell M, Gluckman PD, Harding JE, Challis JRG 2004 Periconceptional undernutrition in sheep accelerates maturation of the fetal hypothalamic-pituitary-adrenal axis in late gestation. Endocrinology 145:4278-4285

46. Fowden AL, Szemere J, Hughes P, Gilmour RS, Forhead AJ 1996 The effects of cortisol on the growth rate of the sheep fetus during late gestation. J Endocrinol 151:97-105

47. Freemark M, Handwerger S 1984 Ovine placental lactogen stimulates glycogen synthesis in fetal rat hepatocytes. Am J Physiol 246:E21-E24
48. Freemark M, Handwerger S 1985 Ovine placental lactogen inhibits glucagon-induced glycogenolysis in fetal rat hepatocytes. Endocrinology 116:1275-1280

49. Bassett NS, Oliver MH, Breier BH, Gluckman PD 1990 The effect of maternal starvation on fetal plasma insulin-like growth factor 1 concentrations in the late gestation ovine fetus. Pediatr Res 27:401-404

50. Gallaher BW, Breier BH, Oliver MH, Harding JE, Gluckman PD 1992 Ontogenic differences in the nutritional regulation of circulating IGF binding proteins in sheep plasma. Acta Endocrinol (Copenh) 126:49-54

51. Baxter RC 1995 Insulin-like growth factor binding proteins as glucoregulators. Metabolism 44:12-17

52. Hawkins P, Steyn C, McGarrigle HH, Calder NA, Saito T, Stratford LL, Noakes DE, Hansona MA 2000 Cardiovascular and hypothalamic-pituitary-adrenal axis development in late gestation fetal sheep and young lambs following modest maternal nutrient restriction in early gestation. Reprod Fertil Dev 12:443-456 